Check for updates

Cite this: RSC Adv., 2017, 7, 49684

\title{
Enhancement of isolation sensitivity for the viable heterogeneous circulating tumor cells swelled by hypo-osmotic pressure $\uparrow$
}

\begin{abstract}
Jiyoon $\mathrm{Bu}^{a}{ }^{a}$ Young-Ho Cho (D) *a and Sae-Won Han ${ }^{\mathrm{b}}$
We present a viable circulating tumor cell (CTC) isolation method based on hypo-osmotic swelling, which is applicable to various size-based CTC isolation devices. The previous CTC filtration devices suffered from the size overlap between CTCs and large leukocytes. Affinity-based size enhancement has been employed to separate CTCs and leukocytes with similar sizes, but the size enhancement was confined to the CTCs expressing specific surface proteins and the cell loss or viability reduction was inevitable when detaching the antibody-conjugated beads from the captured CTCs. In contrast, hypo-osmotic swelling is applicable regardless of the cancer cell types. The size increments of both epithelial- and mesenchymallike cancer cells were larger than that of leukocytes, with less than $10 \%$ of cell death at the osmolality of 190 mOsm kg ${ }^{-1}$. Consequently, cancer cell isolation was 1.2-fold enhanced with negligible reduction in specificity or cell viability, when using one of the conventional CTC filters. We further explored the improvements in CTC isolation using patients' blood samples and confirmed that the CTC detection rate was enhanced when the samples were processed under hypotonic conditions. Our label-free cell size increment technique can be widely applied to the various CTC filters, for enhancing the isolation of

heterogeneous CTCS.
\end{abstract}

Received 21st August 2017
Accepted 14th October 2017

DOI: 10.1039/c7ra09212a

rsc.li/rsc-advances

\section{Introduction}

Metastasis is the leading cause of death among cancer patients, since over $90 \%$ of human deaths due to cancer are strongly associated with metastasis. ${ }^{1}$ Tumour metastasis involves a series of processes, including invasion of a proliferative tumour into the surrounding blood vessels, intravasation of the tumour cells into the blood stream, and the secondary tumour formation after extravasation of the detached tumour cells. ${ }^{2}$ Circulating tumour cells (CTCs) are the cells that have been detached from the primary tumour and circulate through the peripheral blood during the metastasis. It has been reported that the number and the characteristic of CTCs highly match with the malignancy of a tumor. ${ }^{3,4}$ In this regard, CTCs have huge potential as novel biomarkers that not only allow early diagnosis of a tumour but also provide evaluation of treatment effectiveness. $^{5}$

A number of CTC isolation methods have been developed by the advance in microfluidics. Two of the most widely accepted CTC isolation methods are the method based on marker-

${ }^{a}$ Cell Bench Research Center, Korea Advanced Institute of Science and Technology (KAIST), Daejeon 34141, Republic of Korea.E-mail: nanosys@kaist.ac.kr

${ }^{b}$ Department of Internal Medicine, Seoul National University Hospital, Seoul, Republic of Korea

$\dagger$ Electronic supplementary information (ESI) available. See DOI: 10.1039/c7ra09212a specific binding and the method utilizing the difference in size or other physical properties between CTCs and other blood cells. ${ }^{6}$ The affinity-based CTC isolation method relies on the binding force between antibody and specific antigen, which is widely expressed on the surface of CTCs. The only FDA-approved CTC detection system, CellSearch®, also enumerates CTCs using the antibody against epithelial cell adhesion molecule (EpCAM). A number of devices, following CellSearch ${ }^{\circledR}$ system, have immobilized anti-EpCAM antibodies on the surface of various microfluidic devices and successfully isolated cancer cells that show high-EPCAM expressions. ${ }^{7-12}$ However, considering that EpCAM expression is down-regulated after cells experience epithelial-tomesenchymal transition (EMT), the method using antiEpCAM antibody might miss some of the CTC subtypes that are more invasive and directly related to the metastasis. ${ }^{13}$ Other antibodies have been suggested to target these malignant CTC subtypes, including vimentin, MUC1, and CAV1. ${ }^{\mathbf{1 4 - 1 6}}$ These antibodies are also coated on the microfluidic devices, in order to replace or to supplement anti-EpCAM antibody. However, these alternative antibodies either bind with leukocytes or show only a slight improvement. Furthermore, most of the marker-specific isolation methods require long incubation time for the antibody conjugation and show low-throughput; thus, the method is inappropriate for the rapid and reliable tumour diagnosis. 
Label-free methods for isolating CTCs have been developed to overcome the problems associated with the affinity-based isolation. These methods take advantage of difference in physical properties between CTCs and other blood components. Larger size and higher stiffness are two of the most distinct physical properties of CTCs, when compared with the other blood cells. ${ }^{6,17}$ Based on these differences, membrane filters for the size-selective capture $^{18-22}$ and microfluidic channels for the size-based separation $^{23-25}$ have been proposed to isolate CTCs from human blood samples. These devices can be operated at high flow rate and require only simple experimental settings. Most importantly, the label-free isolation is applicable to various CTC subtypes, regardless of the amount of specific surface protein expressions. However, the method has an innate limitation; either sensitivity or purity is comparably lower than that of the affinity-based capture, due to the size overlap between CTCs and large leukocytes.

Recently, novel techniques have been introduced to reduce the bias associated with the size overlap between CTCs and leukocytes. One of the methods is the concurrent use of markerspecific and label-free isolation. ${ }^{26-28}$ Prior to the isolation, nanoparticles or microspheres that are immobilized with anti-EpCAM antibodies are incubated with the blood samples, to amplify the size of CTC-like cells. CTCs were then captured by the sizeselective isolation devices. ${ }^{26,27}$ As a result, these methods have improved the CTC recovery rate and reduced the leukocyte contamination by widening the size difference between CTCs and other blood components. However, the size amplification was limited to the certain type of CTCs; the cells that show highEpCAM expressions. Furthermore, the affinity-based size enhancement lost advantages of size-based filtration method, since it requires complex pre-treatments to attach microspheres on the cells. The chemical damages are also inevitable when detaching microspheres from the captured CTCs.

We propose a novel label-free method based on the hypoosmotic cell swelling, for widening the size difference between CTCs and large leukocytes. Hypo-osmotic swelling has been applied for purifying the mesenchymal stem cells from the body fluid, since these cells are tolerable against the changes in osmotic gradients and show remarkable size enhancement compared to the other blood components. ${ }^{29,30}$ In this respect, the method can also be applied for heterogeneous CTC isolation, since hypo-osmotic pressure not only has a potential to increase the size of various CTC subtypes, but also cause minimal damages on cancer cells when the osmolality is wellcontrolled. Another important advantage of the present method is that the cell swelling generally occurs within several minutes; ${ }^{31}$ thus, unlike the previous immune-specific size amplification, time-consuming pre-treatments are no longer necessary. Therefore, hypo-osmotic cell swelling is expected to improve the capture sensitivity of various size-selective CTC isolation devices, without losing their unique advantages.

\section{Materials and methods}

\section{Hypotonic solution preparation}

Four different hypotonic solutions were prepared by simply mixing the deionized water and $1 \times$ phosphate buffered saline
(PBS) solution (Sigma chemicals, $\sim 280 \mathrm{mOsm} \mathrm{kg}^{-1}$ ) in the ratio of $1: 3,1: 2,1: 1$, or $2: 1$, respectively. As a result, hypotonic buffer solutions having osmolality of 210,190,140, or 95 mOsm $\mathrm{kg}^{-1}$ were produced. The optimal osmolality for the viable CTC isolation was determined by comparing the viability of MCF-7 cancer cells, after exposing the cells on each of different osmolality conditions for $30 \mathrm{~min}$.

\section{Cancer cell model preparation}

Human breast cancer cell line MCF-7, which is well-established epithelial-like CTC (ECTC) model, was donated from Ewha Medical Research Institute. The cells were maintained in RPMI 1640 culture medium (Invitrogen), supplemented by $10 \%$ (v/v) fetal bovine serum (Gibco) and 1\% (v/v) penicillin-streptomycin (Invitrogen), and grown in humidified atmosphere $(5 \%$ $\mathrm{CO}_{2}$ ) at $37{ }^{\circ} \mathrm{C}$. Cells were passaged at $70 \%$ confluence until passage three or four. In order to make mesenchymal-like CTC (MCTC) model, EMT was induced by culturing MCF-7 cells in

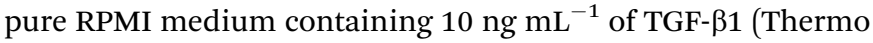
Scientific), an established inducer of EMT. We quantified the mRNA levels of EMT-related markers of ECTC and MCTC models, prior to the experiments (ESI S1†). Both ECTC and MCTC models were cultured for $48 \mathrm{~h}$ and harvested right before the experiments.

\section{Leukocytes preparation}

Leukocytes were collected by following the conventional FicollPaque Plus (GE Healthcare Bio-sciences) density gradient method. In brief, blood samples, obtained from the healthy volunteers, were diluted with PBS solution in the ratio of $1: 2$. The diluted samples were carefully laid above $3 \mathrm{~mL}$ of FicollPaque Plus solution. Subsequently, peripheral blood mononuclear cells (PBMCs) were gently harvested after the centrifugation at $3000 \mathrm{rpm}$ for $20 \mathrm{~min}$, without any break or acceleration. Further centrifugation was conducted at $2500 \mathrm{rpm}$ for $25 \mathrm{~min}$ to collect the leukocytes. Supernatant was removed and leukocytes were contained in the identical volume of PBS solution to that of the original blood sample. All human blood samples were obtained after KAIST institution review board (IRB) approval.

\section{Cell viability analysis}

The viability of breast cancer cells and leukocytes was measured based on two-colored live/dead viability assay (Invitrogen). Cancer cells were harvested from the original culture flask and gently moved to $1.5 \mathrm{~mL}$ tube, at the concentration of approximately $5.0 \times 10^{4}$ cells per mL. Leukocytes were also diluted with PBS solution to a similar concentration. Cells were collected at $3000 \mathrm{~g}$ for $3 \mathrm{~min}$ and the supernatant was removed. Hypoosmotic pressure was induced by resuspending the cells in hypotonic buffer solution. Another centrifugation was conducted after 15 or $30 \mathrm{~min}$ of exposure in hypotonic condition and cells were stained with live/dead double staining kit, which consists of $4.0 \mu \mathrm{M}$ of fluorescein isothiocyanate (FITC) conjugated calcein acetoxymethyl (calcein AM) and tetramethylrhodamine (TRITC) conjugated ethidium homodimer (ET) 
in PBS solution. Cells were collected again by centrifugation at $3000 \mathrm{~g}$ for $3 \mathrm{~min}$ after $20 \mathrm{~min}$ of live/dead staining and the staining solution was replaced with $1 \mathrm{~mL}$ of PBS solution. Viability was measured after loading $80 \mu \mathrm{L}$ of the final sample into each chamber of 96-well plate. The optimal osmolality for the viable CTC isolation was determined by analysing the cell viability.

\section{Cell size measurement}

Changes in cell size was measured by two different methods: (1) changes in cell volume (or diameter) were measured using automated Scepter 2.0 cell counter (Millipore) and (2) changes in surface area were evaluated based on MetaMorph software (Molecular Devices). In case of measuring the changes in cell volume via cell counter, cells were initially contained in $1 \mathrm{~mL}$ of PBS solution. The volume distribution was measured for 0 to $30 \mathrm{~min}$ at different intervals (for every $30 \mathrm{~s}$ until $1 \mathrm{~min}$, for every $1 \mathrm{~min}$ between 1 to $6 \mathrm{~min}$, for every $2 \mathrm{~min}$ between 6 to $10 \mathrm{~min}$, and for every $5 \mathrm{~min}$ between 10 to $30 \mathrm{~min}$ ). The average volume was calculated for each time period, by continuously measuring the cell volume distribution from the same group. The average volume was normalized by comparing with the initial volume. The standard deviation of the normalized values was calculated by analysing the differences in the normalized values, which were obtained from more than three different groups for each cell type $(n>3)$.

Meanwhile, changes in surface area were calculated after harvesting and seeding the cells on 24-well plate with $200 \mu \mathrm{L}$ of PBS solution. After an hour of incubation to settle cells down on the plate, deionized water was gently infused through the chamber at the optimal ratio. The images of swollen cells were taken at different intervals for $30 \mathrm{~min}$ (every $10 \mathrm{~s}$ until $1 \mathrm{~min}$ and every 1 or $2 \mathrm{~min}$ until $30 \mathrm{~min}$ ). Total surface area occupied by the cells was calculated based on MetaMorph image processing.

\section{Model sample preparation}

Model samples consist of approximately 25 cancer cells in $1.5 \mathrm{~mL}$ of either blood or PBS solution, which have been diluted at the optimal ratio with deionized water (hypotonic condition) or PBS solution (isotonic condition). Prior to the isolation, cancer cells were stained with CellTracker green (Molecular Probes, Inc.) by following the manufacturer's instructions.

\section{Human blood sample preparation}

Approximately $2 \mathrm{~mL}$ of whole blood was collected from six colorectal cancer patients by Seoul National University Hospital (SNUH), after IRB approval and patients' agreements. Meanwhile, the same amount of four blood samples were obtained from healthy volunteers with KAIST IRB approval. Blood samples were stored in ethylenediaminetetraacetic acid (EDTA) tubes (Vacuette, greiner bio-one) to prevent the coagulation. Likewise in preparing model samples, $1 \mathrm{~mL}$ of human blood samples were diluted with either deionized water (hypotonic condition) or PBS solution (isotonic condition) at the optimal ratio determined by the previous experiments.

\section{CTC isolation}

Cancer cells were isolated using our mass-producible fabric filters, which have been recently published. ${ }^{\mathbf{1 8}}$ Details are shown in ESI S2 $\dagger$ and in our previous work. ${ }^{18}$ In brief, the fabric filters have slots of $7.6 \pm 1.8 \mu \mathrm{m}$ (warp to warp) $\times 48.2 \pm 2.9 \mu \mathrm{m}$ (weft to weft), which is smaller than the average diameter of cancer cells used in our study (approximately 12.7 $\mu \mathrm{m}$ and $13.7 \mu \mathrm{m}$ for ECTCs and MCTCs, respectively). For the cancer cell isolation, we first infused $1 \mathrm{~mL}$ of optimal hypotonic buffer solution (PBS solution diluted with deionized water) through the filter. Then, the model samples or human blood samples were filtered through our device at the flow rate of $25 \mathrm{~mL} \mathrm{~h}^{-1}$. Prior to the isolation, cells were pre-incubated in hypotonic condition for $6 \mathrm{~min}$, to widen the size difference between cancer cells and leukocytes. After processing the samples, we additionally processed $2.5 \mathrm{~mL}$ of hypotonic buffer solution through the filter, to eliminate the remaining leukocytes and erythrocytes. The captured cells were then released from the filter by manually infusing $4 \mathrm{~mL}$ of trypsin in the reverse direction. The retrieved samples were diluted with $8 \mathrm{~mL}$ of PBS solution and collected after 3 min of centrifugation. Cells were kept in $200 \mu \mathrm{L}$ of fresh PBS solution. Total time required for isolating and collecting the cancer cells (including retrieval and centrifugation process) from $1 \mathrm{~mL}$ of whole blood was less than $22 \mathrm{~min}$. In case of control experiments, which are processed in isotonic condition, PBS solution was used instead of hypotonic buffer solution for the entire process.

\section{Characterization of CTC isolation}

The capture sensitivity was measured by comparing the number of cancer cells that have clogged into the filter, with the initial number of cancer cells spiked into the model samples. The viability of the retrieved cancer cells was analyzed based on twocolored live/dead viability assay. In case of viability measurement, the initial number of cells in model samples were $1 \times 10^{3}$ and the cells were unstained with any fluorescent marker. The leukocyte contamination was measured after processing $1 \mathrm{~mL}$ of human whole blood samples obtained from healthy donors. The retrieved cells were attached to the microscope slides by cyto-spin and the number of leukocytes was counted after immunofluorescence staining. Cells that show positive to both CD45 and DAPI are counted as leukocytes.

\section{Immunofluorescence analysis for CTC enumeration}

The retrieved cells were attached to the microscope slides by cyto-spin. Cells were fixed by $1 \%$ paraformaldehyde (Santa Cruz Biotechnology) for $20 \mathrm{~min}$ and washed with PBS solution. Permeabilization was conducted by dipping the microscope slides in $0.5 \%$ Triton X-100 solution for $30 \mathrm{~min}$. The slide glasses were then immersed in a blocking solution, containing $3 \%$ of bovine serum albumin (Sigma chemicals) in PBS buffer, to prevent the non-specific binding of the leukocytes. Cells were stained for an hour, with FITC-conjugated anti-cytokeratin (15 : 1000 diluted in PBS, BD Biosciences), PE-conjugated mouse anti-human CD45 (20: 1000 diluted in PBS, BD PharmingenTM), Cy5- 
conjugated anti-EpCAM (15: 1000 diluted in PBS, BioLegend) and 4',6-diamidino-2-phenylindole (DAPI, $10: 1000$ diluted in PBS). Finally, microscope slides were washed with fresh PBS solution. CTCs were counted by analyzing the images taken from the microscope. The cells expressing cytokeratin ${ }^{+} / \mathrm{DAPI}^{+} /$ $\mathrm{CD}^{-} 5^{-}$and having larger size than the average size of the background leukocytes were counted as CTCs. ${ }^{32 a}$ Among these CTCs, the cells showing significant expression of EpCAM are considered as EpCAM positive or epithelial-like CTCs while others were counted as CTCs with weak EpCAM expressions or mesenchymal-like subtypes. For the details, we have referred to the criteria of the previous study with a little modification in the intensities. ${ }^{32 b}$ CTCs expressing EpCAM intensity higher than $130 \%$ of the background is regarded as EpCAM positive CTCs while others were counted as EpCAM-weak CTCs.

\section{Results and discussion}

\section{The optimal osmolality for the viable CTC isolation}

Fig. 1 describes the overall experimental scope of the present study. We first compared the viability of MCF-7 cells, to determine the optimal osmolality for the viable CTC isolation. Viability of MCF-7 cells was observed after 15 and $30 \mathrm{~min}$ of exposure in four different hypotonic solutions, having osmolality of 210, 190, 140, and $95 \mathrm{mOsm} \mathrm{kg}^{-1}$, respectively (Fig. 2a and $b$ ). There was no significant reduction in viability at the osmolality above $140 \mathrm{mOsm} \mathrm{kg}^{-1}$. Cell viabilities were $94.9 \pm$ $2.8 \%, 90.5 \pm 1.3 \%$, and $87.9 \pm 3.3 \%$ after inducing hypoosmotic pressure for $30 \mathrm{~min}$ at the osmolality of 210,190 , and $140 \mathrm{mOsm} \mathrm{kg}^{-1}$, respectively. However, only $62.8 \pm 4.0 \%$ of cells remained alive when the osmolality was reduced to 95 mOsm kg ${ }^{-1}$.

Similar results have been reported in the previous studies. It has been widely known that the majority of mammalian cells can withstand the hypo-osmotic gradient above the certain osmolality. Hampton et al. proved that the cells can tolerate the hypo-osmotic pressure, at the osmolality ranging from 310 to 165 mOsm $\mathrm{kg}^{-1} \cdot{ }^{33}$ Kippenberger et al. have verified that there was no significant proof of cell death when the cells were maintained in hypotonic medium with osmolality of 200 mOsm kg ${ }^{-1} \cdot{ }^{34}$ However, cancer cells were unable to tolerate when the osmolality was reduced up to $22 \%$ of the isotonic condition. ${ }^{35}$ Groulx et al. have shown that the most of the cancer cells burst before $15 \mathrm{~min}$ at extreme (98\%) hypotonic condition, while tolerating $50 \%$ of hypotonicity. ${ }^{36}$ Therefore, we conclude that the osmolality should not exceed $140 \mathrm{mOsm} \mathrm{kg}^{-1}$ for the viable CTC isolation. We chose to isolate cancer cells under osmolality of $190 \mathrm{mOsm} \mathrm{kg}{ }^{-1}$, to ensure the cell viability over $90 \%$.

We further extended cell viability experiments to MCTCs and leukocytes. Viabilities of MCTCs and leukocytes were $95.5 \pm$ $2.0 \%$ and $88.0 \pm 2.2 \%$, respectively, when the cells were maintained for $30 \mathrm{~min}$ in hypotonic solution, having osmolality of 190 mOsm kg ${ }^{-1}$ (Fig. 2c). We observed a slight difference between the viabilities of ECTCs and MCTCs. MCTCs show higher resistance to the osmotic stress compared to the epithelial cancer cells. In addition, viability of cancer cells was higher than that of leukocytes.
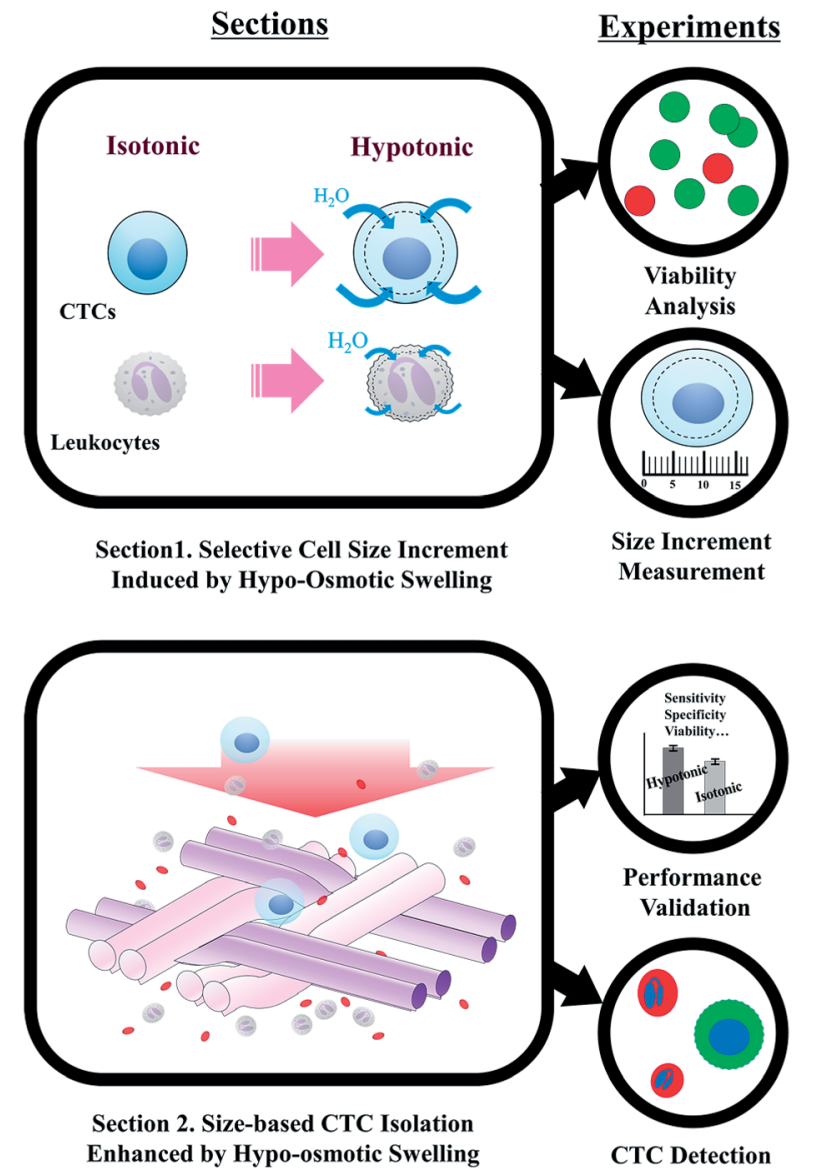

Fig. 1 Experimental procedure for highly-sensitive viable circulating tumor cell isolation based on hypo-osmotic swelling.

Hypo-osmotic pressure has been applied for separating mesenchymal cells from human body fluids, since the cells tolerate well under hypotonic solution. ${ }^{29,30}$ It has been

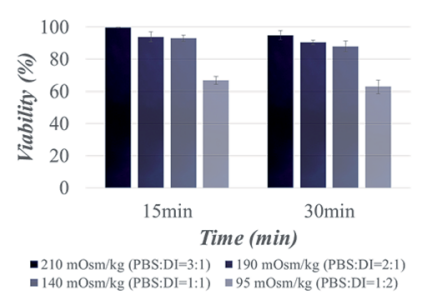

(a)
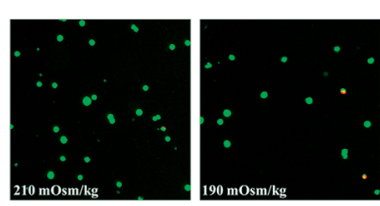

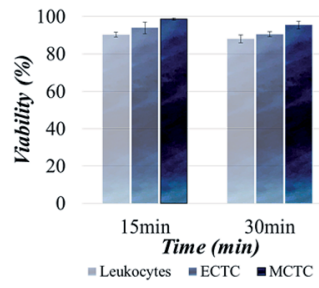

(c)
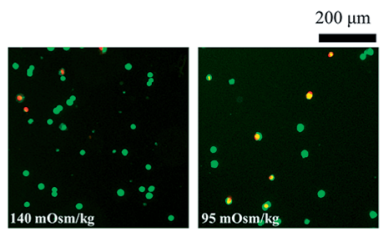

(b)
Fig. 2 Viability of cancer cells and leukocytes after hypo-osmotic swelling: (a) viability of MCF-7 cells depending on the osmolality; (b) two-colored live/dead assay showing the viability of MCF-7 cells depending on the osmolality; (c) viability of cancer cells and the leukocytes in hypotonic solution having osmolality of $190 \mathrm{mOsm} \mathrm{kg}{ }^{-1}$. 
demonstrated that 30 min of exposure in hypotonic condition do not influence neither proliferation ability nor viability of mesenchymal cells. ${ }^{29,30}$ In this manner, we can estimate that the hypotonic solution can also be applied for isolating CTCs, especially the mesenchymal-like CTCs which were difficult to be isolated with the previous CTC isolation methods.

\section{Changes in cell size under hypotonic condition}

We measured the changes in volume and surface area of three different cell types, after inducing hypotonic pressure for $30 \mathrm{~min}$. The average cell volume was measured using automated cell counter. The volume of both ECTCs and MCTCs increased rapidly compared to the leukocytes at the optimal hypotonic condition (Fig. 3a). Cancer cells swelled rapidly until 2-4 min, maintained their volume for the next several minutes, and shrank gradually after $15 \mathrm{~min}$. In contrast, the volume of leukocytes increased only $46.4 \%$ for the first $2 \mathrm{~min}$, which was much lower than either of CTC models (58.8\% for ECTCs and
$67.5 \%$ for MCTCs). Moreover, the maximum volume increase of leukocytes was also smaller than that of cancer cells during $30 \mathrm{~min}$ of exposure in hypotonic condition $(60.4 \%, 68.9 \%$, and $49.6 \%$ for ECTCs, MCTCs, and leukocytes, respectively). The differences in normalized volume changes between cancer cells and leukocytes were confirmed statistically by calculating the differentiation indices (DIs). ${ }^{37}$ DIs for the normalized volume changes between both ECTC-leukocytes and MCTC-leukocytes were higher than 1 for 0 to $15 \mathrm{~min}$, implying that the cancer cells are highly distinguishable from leukocytes by swelling more rapidly and larger at the given osmolality (ESI S3 $\dagger$ ).

It is important to compare the diameters when filtering the cells or small particles, since the filters are generally designed to capture cells or particles having diameters larger than the slot size. Hence, the changes in volume were modified into the diameter as shown in ESI S4. $\dagger$ One of the experimental data showing changes in cell diameter distribution for each cell type is given as an example in Fig. 3b. Even when calculated in terms

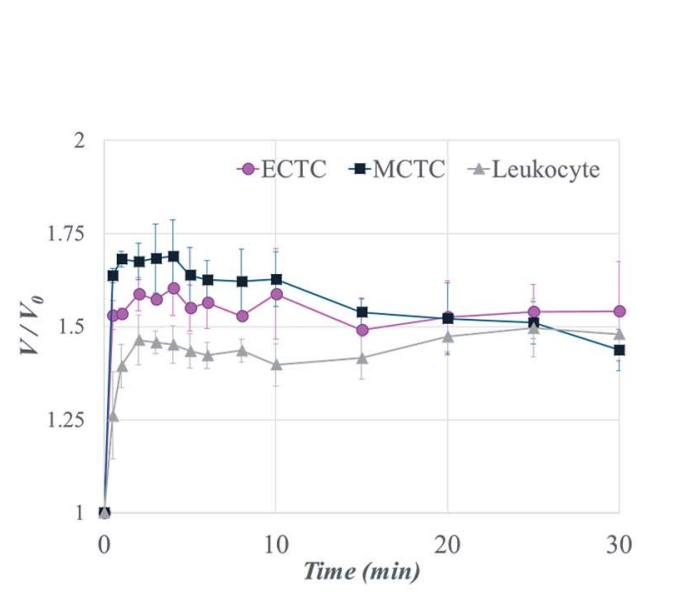

(a)
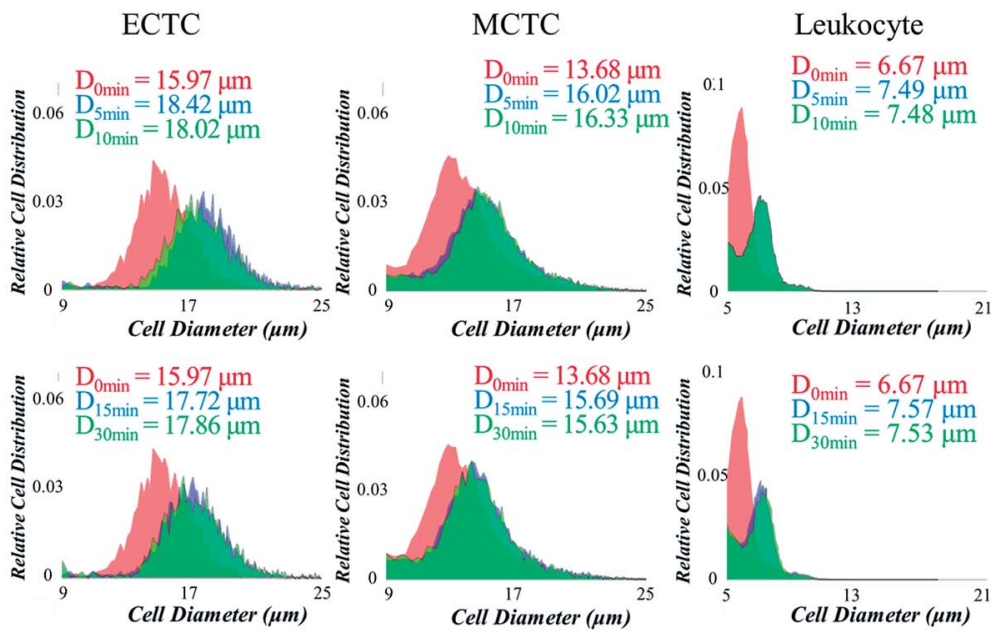

(b)

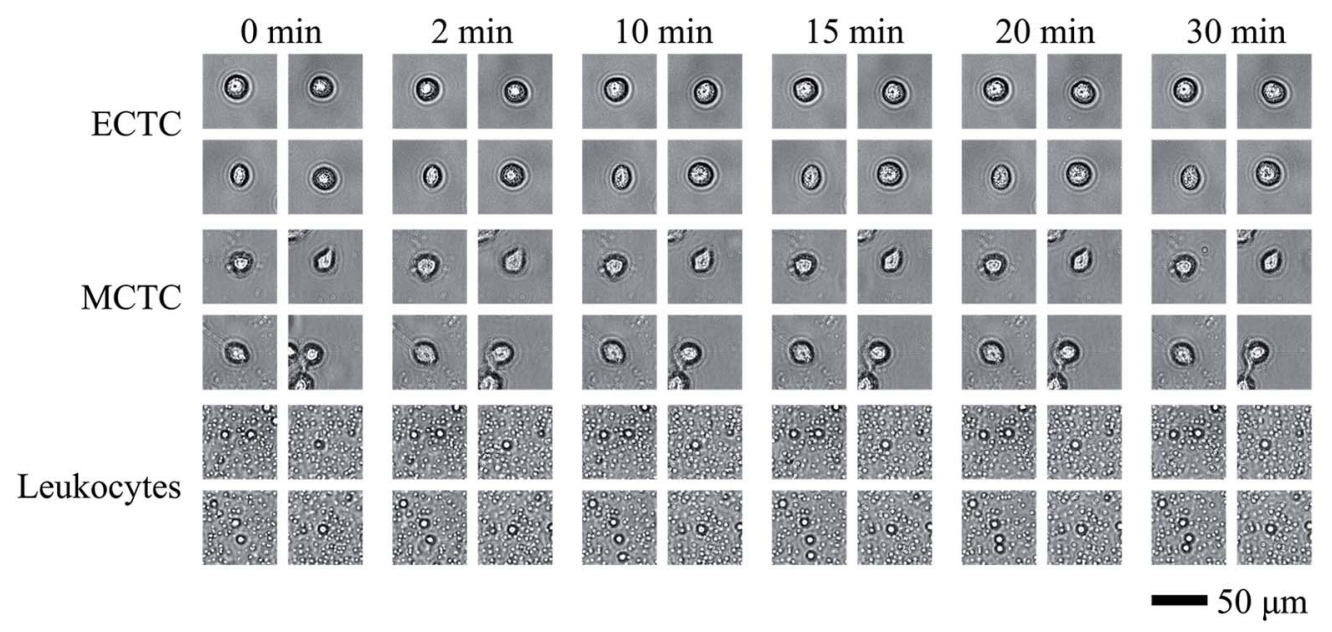

(c)

Fig. 3 Changes in cell size due to hypo-osmotic swelling at the osmolality of $190 \mathrm{mOsm} \mathrm{kg}{ }^{-1}$ : (a) changes in cell volume depending on time; (b) changes in cell diameter depending on time (top: 0-10 min and bottom: 0-30 min); (c) changes in cell surface area depending on time. 
of diameter, cancer cells showed larger size increment compared to the leukocytes, with the maximum differences of $6.0 \%$ and $9.7 \%$ for ECTCs and MCTCs, respectively. We further analyzed the changes of cell size in terms of surface area, by acquiring and analyzing the images of swollen cells for $30 \mathrm{~min}$. Acquired images are given in Fig. $3 \mathrm{c}$ and the details are provided in ESI S5. $\uparrow$ This result also support that the cancer cells swell more rapidly compared to the leukocytes; thus, suggesting that the hypo-osmotic gradients have potential to enhance the sensitivity of CTC capture by increasing the size difference between CTCs and large leukocytes.

It has been extensively known that the cell volume is critical factor for maintaining the cellular functions. ${ }^{38}$ Initially, when the hypo-osmotic pressure is applied to the mammalian cells, uptake of water across the cellular membrane results in cell swelling. The majority of mammalian cells swell in hypotonic condition, due to their large plasma membrane reserves and compensatory exocytosis. ${ }^{36,39}$ Leukocytes, having near-spherical shapes and smaller initial volume than the cancer cells, consist of smaller membrane reserves; therefore, cell swelling is more limited. ${ }^{36}$ However, after certain amount of time, cells stop to swell and starts to shrink due to the process called regulatory volume decrease (RVD), in which swollen cells shrink by releasing potassium and chloride ions with other osmolytes and water. ${ }^{31}$ Cells that are more likely to survive under hypo-osmotic solution, MCTCs in case of our study, tend to overcome the osmotic stress more easily by shifting to their original phase. Due to the RVD, difference in the normalized volume changes between cancer cells and leukocytes diminishes after $15 \mathrm{~min}$. Therefore, to take an advantage of selective cell size amplification, CTC isolation should be performed before $15 \mathrm{~min}$ of exposure at the osmolality of $190 \mathrm{mOsm} \mathrm{kg} \mathrm{kg}^{-1}$. Most of the current high-throughput CTC filtration devices are operated at the flow rate between 10 and $225 \mathrm{~mL} \mathrm{~h}^{-1} \cdot{ }^{\mathbf{1 8 - 2 2 , 4 0 , 4 1}}$ Considering that the cancer cells reach over $90 \%$ of their maximum volume before $3 \mathrm{~min}$ of exposure at the osmolality of $190 \mathrm{mOsm} \mathrm{kg}^{-1}$, $12 \mathrm{~min}$ is sufficient for processing $1 \mathrm{~mL}$ of human blood samples, even when diluted with deionized water at the ratio of $2: 1$. However, when processing larger amount of samples or using filters with lower throughput, pre-treatment with potassium channel blockers or processing at the lower temperature can diminish the effect of RVD and maintain the size difference between CTCs and leukocytes. ${ }^{36,42}$

\section{CTC isolation enhanced by hypo-osmotic swelling}

We first compared the CTC capture sensitivity, with and without inducing hypotonic pressure, in order to clarify that the cell swelling enhances CTC isolation. We used our mass-producible fabric filters as representative CTC filtration devices, which have been recently developed for the viable CTC isolation. ${ }^{18}$ In our previous study, we have succeed in isolating viable CTCs with high throughput $\left(25 \mathrm{~mL} \mathrm{~h}^{-1}\right)$; however, the sensitivity was comparably low by showing capture efficiency of $60-65 \%$ at the given flow rate. ${ }^{\mathbf{1 8}}$

Fig. 4a and b show the capture sensitivity enhanced by hypoosmotic swelling, when the cells were spiked into PBS solution.

Capture sensitivity for ECTCs was 1.18 -fold enhanced by hypoosmotic gradients $(71.9 \pm 4.2 \%$ vs. $60.8 \pm 8.0 \% ; p=0.061)$. Likewise in MCTC models, capture sensitivity was also 1.20-fold increased after hypo-osmotic swelling $(71.3 \pm 4.5 \%$ vs. $59.5 \pm$ $7.5 \% ; p=0.057)$. $P$-Values were near 0.05 for both cell types, indicating that there is a meaningful difference in between two conditions.

Similarly, the capture sensitivity has also increased when the cancer cells were spiked into human blood samples, instead of PBS solution (Fig. 4c). In this case, we spiked cancer cells into the mixture of $1 \mathrm{~mL}$ of human whole blood and $0.5 \mathrm{~mL}$ of deionized water. Cells were kept in mixed solution for $6 \mathrm{~min}$, to widen the size difference between cancer cells and leukocytes. In case of control experiments (isotonic condition), human blood samples were diluted with PBS solution, instead of deionized water. The capture sensitivities for both ECTCs $(67.4 \pm 0.7 \%$ vs. $59.3 \pm 8.6 \% ; p=0.144)$ and MCTCs $(66.6 \pm$ $6.4 \%$ vs. $56.9 \pm 7.7 \% ; p=0.146$ ) have been improved as the cells were processed in hypotonic condition. Although the significance has been weaken, we can still estimate that there is a weak

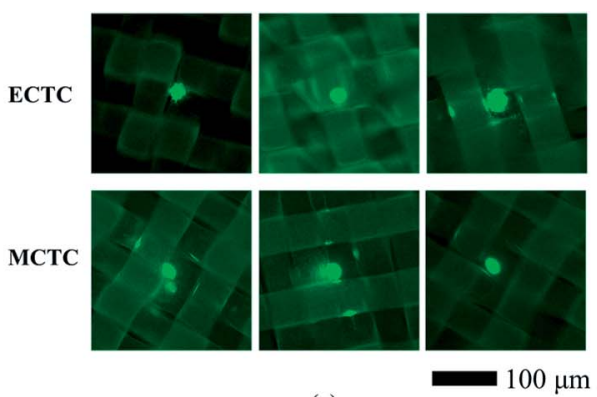

(a)

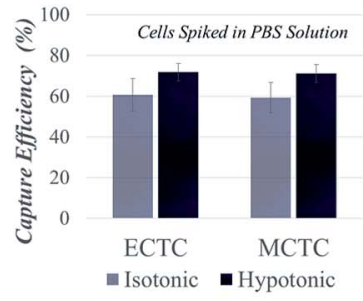

(b)

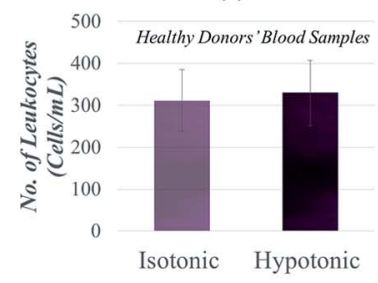

(d)

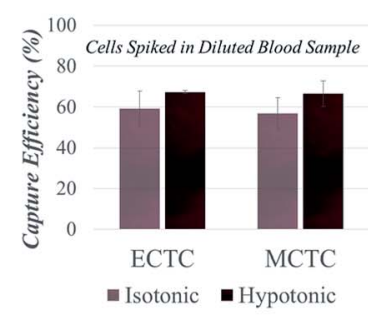

(c)

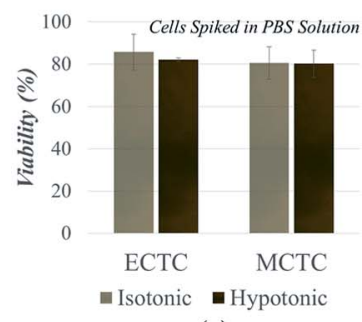

(e)
* Osmolality Condition: Isotonic $=280 \mathrm{mOsm} / \mathrm{kg}$, Hypotonic $=190 \mathrm{mOsm} / \mathrm{kg}$

Fig. 4 CTC isolation enhanced by hypo-osmotic swelling: (a) images of the captured cancer cells; (b) osmolality-dependent capture efficiency of the cancer cells spiked into PBS solution; (c) osmolalitydependent capture efficiency of the cancer cells spiked into human blood obtained from healthy donors; (d) osmolality-dependent leukocyte contamination rate; (e) osmolality-dependent viability of the retrieved cancer cells. 
tendency showing the difference in capture sensitivity $(p<0.15)$. Another important finding was that the hypo-osmotic pressure was beneficial for enhancing the sensitivity of cancer cells, regardless of their phenotype. The previous size amplification method which rely on binding force between antibody and antigen, could not effectively increase the size of the cancer cells that do not express specific surface antigens, sufficiently. ${ }^{26-28}$ In contrast, our method has enhanced the capture sensitivity for both epithelial and mesenchymal cancer cells at the similar ratio (1.13-fold vs. 1.17-fold).

We further analysed the decrease in the specificity, which is mainly due to the size increase of the leukocytes (Fig. 4d). We measured the number of leukocytes in the retrieved samples and calculated the leukocyte depletion ratio, after processing four human blood samples obtained from healthy donors. The average number of the captured leukocytes in $1 \mathrm{~mL}$ of human blood were $330.0 \pm 73.3$ at $190 \mathrm{mOsm} \mathrm{kg}^{-1}$ and $311.5 \pm 77.4$ at the $280 \mathrm{mOsm} \mathrm{kg}^{-1}$, respectively $(p=0.087)$. When considering that the total number of leukocytes in $1 \mathrm{~mL}$ of human whole blood range from 4 to 10 millions, increase in leukocyte contamination due to the hypo-osmotic pressure is negligible. Over $99.9 \%$ of leukocytes were eliminated in all four human blood samples, regardless of the osmolality. Consequently, the specificity reduction was less than $6 \%$ under hypotonic condition, since the leukocytes swell smaller and more slowly than the cancer cells.

For the next step, we confirmed the viability of the isolated cancer cells (Fig. 4e). The experiments were conducted to clarify that the cells are still viable, even after exposed to the combined effect of osmotic and mechanical stresses, which are induced by hypo-osmotic pressure and squeezing during the isolation process, respectively. The viability reduction due to the osmotic pressure was insignificant for both ECTCs $(82.1 \pm 2.1 \% v s$. $85.7 \pm 5.7 \% ; p=0.449)$ and MCTCs $(80.5 \pm 2.5 \%$ vs. $80.2 \pm$ $3.6 \% ; p=0.917)$. The cell viability obtained after the isolation process was highly correlated with the results obtained from the previous experiments. Mesenchymal-like cancer cells, which are more tolerable to the changes in osmolality, showed lower reduction in viability compared to the epithelial cancer cells. However, irrespective to the cell type, the viability reduction induced by the hypo-osmotic pressure show no significance $(p>0.449)$.

Though we have applied hypo-osmotic swelling to our fabric filters only, the method can be broadly applied to various sizebased CTC isolation devices. However, the enhancement in capture sensitivity might differ depending on the size of the slots or the processing conditions. Another important advantage of applying hypo-osmotic pressure during CTC isolation is that the method is beneficial for capturing CTCs with high invasive potentials, which were easily missed with the previous devices or methods. Size-selective isolation methods have higher chances to capture EpCAM down-regulated CTCs compared to the affinity-based approaches, but still suffer from capturing these subtypes. EMT not only induces cells to lose their EpCAM expressions but also meditates cells to possess higher deformability. ${ }^{43}$ As a result, the mesenchymal CTCs have higher potential to escape from the slots. In this regard, hypoosmotic cell swelling can be a preferable option for minimizing the loss of these more invasive CTCs.

\section{Verification of enhancement in CTC isolation using clinical samples}

Clinical application has been demonstrated using ten human blood samples, obtained from six patients with colorectal tumour and four healthy donors, respectively (Fig. 5). Prior to the clinical study, we have demonstrated that our system is also applicable for detecting colorectal CTCs, by using human colorectal cancer cell line SW620 (ESI S6†). Table 1 shows the number of CTCs detected under isotonic and hypotonic conditions, respectively. The clinical data for each patient is supplied in ESI S7. $\uparrow$ The results can be summarized as followed: (1) all six patients' samples showed at least three CTCs, when the blood samples were filtered under hypotonic condition. (2) Meanwhile, none of suspicious CTC-like cells were detected in

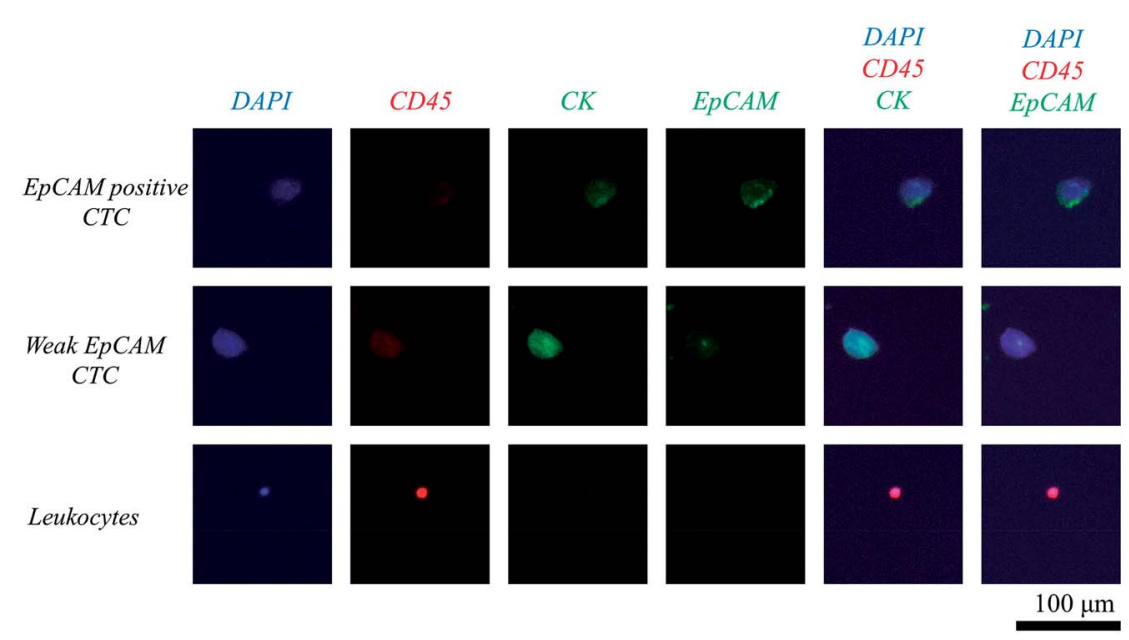

Fig. 5 Fluorescence microscope image of isolated circulating tumor cells and leukocytes stained with DAPI (blue), EpCAM (green), cytokeratin (green), and CD45 (red). 
Table 1 The number of circulating tumour cells detected in human blood samples depending on osmolality

\begin{tabular}{|c|c|c|c|c|c|c|c|}
\hline \multirow[b]{2}{*}{ Sample ID } & \multirow[b]{2}{*}{ Stage/state } & \multicolumn{3}{|c|}{ Hypotonic (190 mOsm kg-1) } & \multicolumn{3}{|c|}{ Isotonic $\left(280 \mathrm{mOsm} \mathrm{kg}^{-1}\right)$} \\
\hline & & Total & EpCAM positive CTCs & EpCAM weak CTCs & Total & EpCAM positive CTCs & EpCAM weak CTCs \\
\hline P2 & $\mathrm{I} /$ recurred & 17 & 13 & 4 & 13 & 11 & 2 \\
\hline P3 & IV/initial & 6 & 5 & 1 & 6 & 5 & 1 \\
\hline $\mathrm{P} 4$ & III/recurred & 3 & 3 & 0 & 2 & 2 & 0 \\
\hline $\mathrm{H} 1^{b}$ & - & 0 & 0 & 0 & 0 & 0 & 0 \\
\hline $\mathrm{H} 2$ & - & 0 & 0 & 0 & 0 & 0 & 0 \\
\hline H3 & - & 0 & 0 & 0 & 0 & 0 & 0 \\
\hline $\mathrm{H} 4$ & - & 0 & 0 & 0 & 0 & 0 & 0 \\
\hline
\end{tabular}

${ }^{a}$ Samples obtained from colorectal cancer patients. ${ }^{b}$ Samples obtained from healthy donors.

the samples obtained from healthy donors. (3) Hypo-osmotic pressure enhances the CTC detection rate, by isolating more or same number of CTCs in all six patients' samples. (4) The improvement in detection rate was not only confined to EPCAMpositive CTCs, but also to CTCs having weak EpCAM expressions. (5) Furthermore, CTC purity in retrieved samples has been enhanced. This implies that despite of increase in the number of leukocytes, the number of CTCs increased more significantly.

Particularly, CTCs were observed in all six colorectal cancer patients' samples in the experiments conducted at the hypotonic condition. Meanwhile, none of CTCs were found in one of the patients' samples (sample P5) under isotonic condition. More or same number of CTCs were isolated in hypotonic condition, without any exception. The enhancement in CTC detection due to the hypo-osmotic cell swelling was statistically significant (8.3 \pm 4.9 CTCs per $\mathrm{mL} v s .5 .6 \pm 4.3$ CTCs per $\mathrm{mL} ; p=0.014$ ).

As described in the previous sections, hypo-osmotic cell swelling is beneficial for improving the detection of EpCAM down-regulated CTCs. This has been proved by enumerating the CTCs with weak EpCAM expressions. EpCAM down-regulated CTCs were found in four of six patients' samples in hypotonic condition, with average number of $1.8 \pm 1.7$ CTCs per mL. Meanwhile, this subtype was detected in only half of the patients' samples in isotonic condition, with the average number of $0.7 \pm 0.7$ CTCs per mL. As a result, the proportion of EpCAM down-regulated CTCs was $18 \%$ in hypotonic condition, which was more than twice higher than that in isotonic condition.

We also calculated the purity in terms of the number of CTCs over the total number of cells retrieved after entire filtration process (ESI S8†). Despite of increase in the number of leukocytes in hypotonic condition, purity was higher for all cases with only one exception (sample P3). The results reveal that the number of isolated CTCs increased more sharply compared to the number of leukocytes. The average purity of CTCs in hypotonic condition was $2.7 \pm 1.3 \%$, which was significantly higher than the control cases, showing CTC purity of $1.9 \pm 1.3 \%$ ( $p=$ 0.011). Therefore, not only the sensitivity, but also the purity for CTC isolation can be enhanced by applying osmotic stress.
The clinical relevance between the number of CTCs and the patients' clinical data was difficult to be found at this moment, since the number of samples were too small to derive the statistical significance. Comparatively large number of CTCs were found among patients with recurrent tumour $(10.7 \pm 7.1$ CTCs per $\mathrm{mL}$ vs. $6.0 \pm 2.0$ CTCs per $\mathrm{mL} ; p=0.334$ ), but the statistical significance was poor. However, we can still expect that our method is applicable for characterizing the possibility of metastasis, since all the patients involved in our study have experienced metastasis and also showed at least three CTCs in $1 \mathrm{~mL}$ of blood samples.

\section{Conclusions}

We have applied hypo-osmotic cell swelling for CTC filtration, in order to enhance the CTC isolation sensitivity by increasing the size difference between cancer cells and large leukocytes. Regardless of their phenotype, cancer cells were highly tolerable to the osmotic gradients. Over $90 \%$ of cancer cells maintained their viability, after $30 \mathrm{~min}$ of exposure at the osmolality of 190 mOsm $\mathrm{kg}^{-1}$. Consequently, $80-82 \%$ of cancer cells remained alive even after the filtration process. The cell swelling was also beneficial for selectively amplifying the size of cancer cells. Depending on the phenotype, cancer cells showed maximum $55-70 \%$ of volume increase during $30 \mathrm{~min}$ of exposure on hypotonic solution, while less than $50 \%$ of size increase was found from leukocytes. As a result, cancer cell capture efficiency was approximately 1.2-fold increased. Clinical study has been demonstrated using ten human blood samples. CTCs were found in all six colorectal cancer patients' samples, while none was detected among healthy volunteers. Both the number of CTCs and CTC detection rate were enhanced by hypo-osmotic swelling. Most importantly, our method was advantageous for detecting the mesenchymal-like CTCs, which were easily missed by the previous CTC isolation methods. Since EMT meditates cancer cells to lose their EpCAM expressions and enhances cell deformability, CTC isolation methods based on both biochemical and biophysical properties suffer from capturing these subtypes. By inducing hypo-osmotic stress to these subtypes, capture efficiency has been highly improved; thus, we 
have succeed in capturing 1-4 CTCs with low EpCAM expressions in six out of four cancer patients.

For the future work, we are planning to extend our study by applying our new method to other size-based CTC isolation devices. Next, we are expecting to find clinical meanings by conducting further analysis on patients' blood samples and increasing the sample numbers. In summary, hypo-osmotic cell swelling enhances the sensitivity of CTC isolation and has potential to enhance the clinical significance of CTC-based cancer research.

\section{Conflicts of interest}

There are no conflicts to declare.

\section{Acknowledgements}

This research was supported by Korea Evaluation Institute of Industrial Technology (Project No. 10078295). The research was also supported by Yoon-Tae Kang of the Cell Bench Research Center, KAIST, who helped searching the previous works and designing the experiments, and Tae Hee Lee and Ji Hye Choi, of the same institution, who helped with experiments.

\section{Notes and references}

1 B. Weigelt, J. L. Peterse and L. J. van't Veer, Nat. Rev. Cancer, 2005, 5, 591-602.

2 N. Reymond, B. B. d'Agua and A. J. Ridley, Nat. Rev. Cancer, 2013, 13, 858-870.

3 M. Spiliotaki, D. Mavroudis, K. Kapranou, H. Markomanolaki, G. Kallergi, F. Koinis, K. Kalbakis, V. Georgoulias and S. Agelaki, Breast Cancer Res., 2014, 16, 485.

4 K. Bluemke, U. Bilkenroth, A. Meye, S. Fuessel, C. Lautenschlaeger, S. Goebel, A. Melchior, H. Heynemann, P. Fornara and H. Taubert, Cancer Epidemiol., Biomarkers Prev., 2009, 18, 2190-2194.

5 J. S. de Bono, H. I. Scher, R. B. Montgomery, C. Parker, M. C. Miller, H. Tissing, G. V. Doyle, L. W. W. M. Terstappen, K. J. Pienta and D. Raghavan, Clin. Cancer Res., 2008, 14, 6302-6309.

6 V. Murlidhar, L. Rivera-Baez and S. Nagrath, Small, 2016, 12, 4450-4463.

7 S. Nagrath, L. V. Sequist, S. Maheswaran, D. W. Bell, D. Irimia, L. Ulkus, M. R. Smith, E. L. Kwak, S. Digumarthy, A. Muzikansky, P. Ryan, U. J. Balis, R. G. Tompkins, D. A. Haber and M. Toner, Nature, 2007, 450, 1235-1239.

8 A. A. Adams, P. I. Okagbare, J. Feng, M. L. Hupert, D. Patterson, J. Gottert, R. L. McCarley, D. Nikitopoulos, M. C. Murphy and S. A. Soper, J. Am. Chem. Soc., 2008, 130, 8633-8641.

9 V. Murlidhar, M. Zeinali, S. Grabauskiene, M. GhannadRezaie, M. S. Wicha, D. M. Simeone, N. Ramnath, R. M. Reddy and S. Nagrath, Small, 2014, 10, 4895-4904.
10 H. J. Yoon, T. H. Kim, Z. Zhang, E. Azizi, T. M. Pham, C. Paoletti, J. Lin, N. Ramnath, M. S. Wicha, D. F. Hayes, D. M. Simeone and S. Nagrath, Nat. Nanotechnol., 2013, 8, 735-741.

11 F. Fachin, G. D. Chen, M. Toner and B. L. Wardle, J. Microelectromech. Syst., 2011, 20, 1428-1438.

12 Y. T. Lu, L. B. Zhao, Q. L. Shen, M. A. Garcia, D. X. Wu, S. Hou, M. Song, X. C. Xu, W. H. OuYang, W. W. L. OuYang, J. Lichterman, Z. Luo, X. Xuan, J. T. Huang, L. W. K. Chung, M. Rettig, H. R. Tseng, C. Shao and E. M. Posadas, Methods, 2013, 64, 144-152.

13 N. M. Karabacak, P. S. Spuhler, F. Fachin, E. J. Lim, V. Pai, E. Ozkumur, J. M. Martel, N. Kojic, K. Smith, P. I. Chen, J. Yang, H. Hwang, B. Morgan, J. Trautwein, T. A. Barber, S. L. Stott, S. Maheswaran, R. Kapur, D. A. Haber and M. Toner, Nat. Protoc., 2014, 9, 694-710.

14 A. Satelli and S. L. Li, Cell. Mol. Life Sci., 2011, 68, 3033-3046. 15 F. I. Thege, T. B. Lannin, T. N. Saha, S. Tsai, M. L. Kochman,

M. A. Hollingsworth, A. D. Rhim and B. J. Kirby, Lab Chip, 2014, 14, 1775-1784.

16 Y. J. Kim, G. B. Koo, J. Y. Lee, H. S. Moon, D. G. Kim, D. G. Lee, J. Y. Lee, J. H. Oh, J. M. Park, M. S. Kim, H. G. Woo, S. I. Kim, P. Kang, W. Choi, T. S. Sim, W. Y. Park, J. G. Lee and Y. S. Kim, Biomaterials, 2014, 35, 7501-7510.

17 J. S. Bagnall, S. Byun, S. Begum, D. T. Miyamoto, V. C. Hecht, S. Maheswaran, S. L. Stott, M. Toner, R. O. Hynes and S. R. Manalis, Sci. Rep., 2015, 5, 18542.

18 J. Bu, Y.-T. Kang, Y.-S. Lee, J. Kim, Y.-H. Cho and B.-I. Moon, Biosens. Bioelectron., 2017, 91, 747-755.

19 S. Y. Zheng, H. K. Lin, B. Lu, A. Williams, R. Datar, R. J. Cote and Y. C. Tai, Biomed. Microdevices, 2011, 13, 203-213.

20 D. L. Adams, P. X. Zhu, O. V. Makarova, S. S. Martin, M. Charpentier, S. Chumsri, S. H. Li, P. Amstutz and C. M. Tang, RSC Adv., 2014, 4, 4334-4342.

21 X. Y. Fan, C. P. Jia, J. Yang, G. Li, H. J. Mao, Q. H. Jin and J. L. Zhao, Biosens. Bioelectron., 2015, 71, 380-386.

22 Y. D. Tang, J. Shi, S. S. Li, L. Wang, Y. E. Cayre and Y. Chen, Sci. Rep., 2014, 4, 6052.

23 H. W. Hou, M. E. Warkiani, B. L. Khoo, Z. R. Li, R. A. Soo, D. S. W. Tan, W. T. Lim, J. Han, A. A. S. Bhagat and C. T. Lim, Sci. Rep., 2013, 3, 1259.

24 R. M. Jack, M. M. G. Grafton, D. Rodrigues, M. D. Giraldez, C. Griffith, R. Cieslak, M. Zeinali, C. K. Sinha, E. Azizi, M. Wicha, M. Tewari, D. M. Simeone and S. Nagrath, Adv. Sci., 2016, 3, 1600063.

25 A. A. S. Bhagat, H. W. Hou, L. D. Li, C. T. Lim and J. Y. Han, Lab Chip, 2011, 11, 1870-1878.

26 J. H. Shin, M. G. Lee, S. Choi and J. K. Park, RSC Adv., 2014, 4, 39140-39144.

27 M. S. Kim, T. S. Sim, Y. J. Kim, S. S. Kim, H. Jeong, J. M. Park, H. S. Moon, S. I. Kim, O. Gurel, S. S. Lee, J. G. Lee and J. C. Park, Lab Chip, 2012, 12, 2874-2880.

28 Q. Q. Huang, B. Cai, B. L. Chen, L. Rao, Z. B. He, R. X. He, F. Guo, L. B. Zhao, K. K. Kondamareddy, W. Liu, S. S. Guo and X. Z. Zhao, Adv. Healthcare Mater., 2016, 5, 1554-1559. 
29 C. B. Lin, M. R. Shen, W. P. Chen, X. F. Li, D. M. Luo, J. H. Cai and Y. Yang, In Vitro Cell. Dev. Biol.: Anim., 2015, 51, 11021108.

30 B. Parekkadan, P. Sethu, D. Van Poll, M. L. Yarmush and M. Toner, Tissue Eng., 2007, 13, 2465-2474.

31 E. K. Hoffmann, I. H. Lambert and S. F. Pedersen, Physiol. Rev., 2009, 89, 193-277.

32 (a) S. Park, R. R. Ang, S. P. Duffy, J. Bazov, K. N. Chi, P. C. Black and H. S. Ma, PLoS One, 2014, 9, e85264; (b) J. Bu, Y. J. Kim, Y. T. Kang, T. H. Lee, J. Kim, Y. H. Cho and S. W. Han, Biomaterials, 2017, 125, 1-11.

33 R. Y. Hampton and R. W. Holz, J. Cell Biol., 1983, 96, 10821088.

34 S. Kippenberger, S. Loitsch, M. Guschel, J. Muller, R. Kauffman and A. Bernd, FEBS Lett., 2005, 579, 207-214.

35 N. Selzner, M. Selzner, R. Graf, U. Ungethuem, J. G. Fitz and P. A. Clavien, Cell Death Differ., 2004, 11, S172-S180.
36 N. Groulx, F. Boudreault, S. N. Orlov and R. Grygorczyk, J. Membr. Biol., 2006, 214, 43-56.

37 Y. Park, H. W. Kim, J. Yun, S. Seo, C. J. Park, J. Z. Lee and J. H. Lee, BioMed Res. Int., 2016, 2016, 8748023.

38 F. Lang, G. L. Busch, M. Ritter, H. Volkl, S. Waldegger, E. Gulbins and D. Haussinger, Physiol. Rev., 1998, 78, 247306.

39 C. E. Morris and U. Homann, J. Membr. Biol., 2001, 179, 79102.

40 H. K. Lin, S. Y. Zheng, A. J. Williams, M. Balic, S. Groshen, H. I. Scher, M. Fleisher, W. Stadler, R. H. Datar, Y. C. Tai and R. J. Cote, Clin. Cancer Res., 2010, 16, 5011-5018.

41 R. Harouaka, Z. G. Kang, S. Y. Zheng and L. Cao, Pharmacol. Ther., 2014, 141, 209-221.

42 H. Pasantes-Morales and S. M. Mulia, Nephron, 2000, 86, 414-427.

43 H. D. Huang, R. D. Kamm and R. T. Lee, Am. J. Physiol., 2004, 287, C1-C11. 